Polymer Journal, Vol. 38, No. 8, pp. 835-843 (2006)

(C) 2006 The Society of Polymer Science, Japan

\title{
In Situ Polymerization and Properties of Methyl Methacrylate-Butadiene-Styrene Resin with Bimodal Rubber Particle Size Distribution
}

\author{
Jun TAKAHAShI, ${ }^{1, \dagger}$ Hideki Watanabe, ${ }^{1}$ Jun NAKAMOTO, ${ }^{1}$ \\ Kazuo AraKawa, ${ }^{2}$ and Mitsugu TODO ${ }^{2}$ \\ ${ }^{1}$ Polymer Research Center, Denki Kagaku Kogyo Kabushiki Kaisha, 6 Goiminamikaigan, Ichihara 290-8588, Japan \\ ${ }^{2}$ Research Institute for Applied Mechanics, Kyushu University, 6-1 Kasugakouen, Kasuga 816-8580, Japan
}

(Received January 6, 2006; Accepted April 24, 2006; Published July 14, 2006)

\begin{abstract}
The in situ polymerization synthesis and properties of methyl methacrylate-butadiene-styrene resin (MBS resin) with bimodal rubber particle size distribution were investigated. MBS resin was prepared by bulk-suspension polymerization of styrene and methyl methacrylate in the presence of the styrene-butadiene block copolymer, and bimodal rubber particle size distribution was observed during bulk polymerization and cooling. During bulk polymerization and cooling, size distribution was regulated by the viscosity of the prepolymer or shearing for the prepolymer. The formative mechanisms of the bimodal distribution were presumed to be increase of small rubber particles by shearing and delay in changing to small rubber particles according to the difference in the inside structure of large rubber particles. MBS resin with the bimodal distribution was obtained by suspension polymerization using the prepolymer with bimodal distribution. The resin was superior with regard to balance of impact resistance and transparency. [doi:10.1295/polymj.PJ2005203]

KEY WORDS In Situ Polymerization / Property / Methyl Methacrylate-Butadiene-Styrene Resin (MBS Resin) / Bimodal Distribution / Rubber Particle Size /
\end{abstract}

Methyl methacrylate-butadiene-styrene resin (MBS resin) has rubber particles dispersed throughout a matrix of methyl methacrylate-styrene polymer. It has good balance of transparency and impact resistance, is widely used as a molding material for household articles, electric appliances and packages. However, more improvement of transparency and impact resistance is desirable.

The MBS resin becomes transparent by making equal the refractive index of the matrix and that of rubber particles. The size of the rubber particles also affects transparency of the MBS resin. If the size of the rubber particles is large, the transparency of the molded article is poor because of the surface is uneven. As transparency is an important quality of the MBS resin, small rubber particles are desirable. However, small rubber particles reduce impact resistance. Therefore, the distribution of the rubber particle size is important for balancing transparency and impact resistance.

MBS resin having both small and large rubber particles, in a bimodal size distribution, shows good transparency and high impact resistance. ${ }^{1-3}$ Although high impact strength polystyrene (HI-PS) is opaque, bimodal distribution of the rubber particle size produces good high impact resistance. ${ }^{4-8}$ These bimodal distributions of the rubber particle size were made by blending two resins or two prepolymers with dif- ferent particle sizes.

This study describes 'in situ' process for making a bimodal MBS resin without blending by a bulk-suspension polymerization of styrene and methyl methacrylate in the presence of the styrene-butadiene copolymer. Bimodal MBS resin obtained by this process had good balance of transparency and impact resistance.

\section{EXPERIMENTAL}

\section{Bulk Polymerizations (runs 1-6)}

A styrene-butadiene block copolymer (670A, Asahi Kasei, styrene content: $40.6 \%)(8 \mathrm{~kg})$ was dissolved in a monomer mixture comprising styrene $(40 \mathrm{~kg})$, methyl methacrylate $(28 \mathrm{~kg})$ and $n$-butyl acrylate $(4 \mathrm{~kg}) . n$-Butyl acrylate was added for the purpose of improving the molding processability of the MBS resin. A mixture $(80 \mathrm{~kg}), t$-butyl peroxy-2-ethyl-hexanoate $(43 \mathrm{~g})$ as polymerization initiator and $t$-dodecylmercaptan $(t-\mathrm{DDM})$ (run $1-4 ; 120 \mathrm{~g}$, run $5 ; 144 \mathrm{~g}$, run $6 ; 168 \mathrm{~g})$ as a chain transfer agent was added to a stirred tank reactor. The mixture was heated at $92^{\circ} \mathrm{C}$ for $7.5 \mathrm{~h}$ with stirring at a selected speed of $100 \mathrm{rpm}$ (run 1), $130 \mathrm{rpm}$ (run 2), $160 \mathrm{rpm}$ (run 3), and $190 \mathrm{rpm}$ (run 4-6) and then cooled to stop the bulk polymerization.

${ }^{\dagger}$ To whom correspondence should be addressed (Fax: +81-436-26-3320, E-mail: jun-takahashi@denka.co.jp). 
Bulk Polymerization and Suspension Polymerizations (run 7)

A styrene-butadiene block copolymer (670A, Asahi Kasei, styrene content: $40.6 \%)(8 \mathrm{~kg})$ was dissolved in a monomer mixture comprising styrene $(40 \mathrm{~kg})$, methyl methacrylate $(28 \mathrm{~kg})$ and $n$-butyl acrylate $(4 \mathrm{~kg})$. The mixture $(80 \mathrm{~kg}), t$-butyl peroxy-2-ethyl-hexanoate $(43 \mathrm{~g})$ and $t$-DDM $(160 \mathrm{~g})$ was added to a stirred tank reactor. The mixture was heated at $92{ }^{\circ} \mathrm{C}$ for $6.5 \mathrm{~h}$ with stirring at a selected speed of $190 \mathrm{rpm}$. The mixture was cooled at $60^{\circ} \mathrm{C}$ for $2 \mathrm{~h}$ and $25^{\circ} \mathrm{C}$ for several hours with stirring at a selected speed of $190 \mathrm{rpm}$. Some prepolymers were sampled during bulk polymerization and cooling (Figure 3).

Distilled water $(7 \mathrm{~kg})$, sodium dodecylbenzene sulfonate $(35 \mathrm{mg})$ and calcium tertiary phosphate $(17.5 \mathrm{~g})$ as suspension stabilizers were added to another stirred tank reactor. One of the prepolymers $(3.5 \mathrm{~kg})$ and ethyl-3,3-di(t-butyl peroxy)butylate $(5.25 \mathrm{~g})$ as a polymerization initiator were dispersed therein with stirring. This mixture was heated at $115^{\circ} \mathrm{C}$ for $6.5 \mathrm{~h}$ and at $130^{\circ} \mathrm{C}$ for $2 \mathrm{~h}$, and the prepolymer was completely polymerized.

After polymerization, the product was washed with distilled water, the water was removed and dried to obtain the MBS resin in the form of beads. The beads were processed into pellets by a single screw extruder (FS40, Ikegai) at a cylinder temperature of $220^{\circ} \mathrm{C}$.

\section{Measurements}

Rubber particle size and distribution were measured with a laser diffraction particle size analyzer (LS230, Beckman Coulter). The measurement solvent was $N, N$-dimethylformamide.

TEM observation was made with a Hitachi H7500. Solid test specimens were stained with osmic acid and sliced into ultrathin sections. As the prepolymer is liquid, the test specimens were sliced into ultrathin sections after encapsulating with epoxy resin.

Viscosity was measured using a Brookfield viscometer (BL, Tokimec) at different temperatures. The measured viscosity was converted to viscosity at the temperature in the stirred tank reactor.

Charpy notched impact strength was measured with a fully automatic Charpy impact tester (258ZA, Yasuda seiki) at $23^{\circ} \mathrm{C}$, as specified by ISO179 and test pieces of $80 \times 10 \times 4 \mathrm{~mm}$ with a V-notch $2 \mathrm{~mm}$ deep. The test pieces were injection molded from pellets by a injection machine (IS50EP, Toshiba kikai) at the cylinder temperature of $220^{\circ} \mathrm{C}$ and the mold temperature of $45^{\circ} \mathrm{C}$, as specified by ISO2897-2.

Haze was measured with a haze meter (NDH2000, Nihon Densyoku) at $23^{\circ} \mathrm{C}$, as specified by JIS (K7105) using test pieces of $40 \times 120 \times 2 \mathrm{~mm}$. The test pieces were injection molded from pellets by a injection ma- chine (IS50EP, Toshiba kikai) at the cylinder temperature of $230^{\circ} \mathrm{C}$ and the mold temperature of $60^{\circ} \mathrm{C}$.

\section{RESULTS AND DISCUSSION}

\section{Distribution of Rubber Particle Sizes in Polymeriza- tions (runs 1-6)}

Rubber particle size distribution in the bulk polymerization was investigated and that of rubber particle size by the rotational speed of $100 \mathrm{rpm}$ (run 1), 130 rpm (run 2), $160 \mathrm{rpm}$ (run 3), and $190 \mathrm{rpm}$ (run 4) is shown in Figure 1. Figure 1a shows size distributions at the end (before cooling) of the bulk polymerization, and Figure 1b shows size distributions after cooling. Size distributions depended on rotational speed in the bulk polymerization in case of before cooling (Figure 1a). Size distributions did not have a remarkable difference owing to rotational speed in case of after cooling (Figure 1b). Each polymer content of runs 1-4 was almost the same (about 35\%), and the polymer content of before cooling and after cooling was unchanged. Polymer content was measured by separating polymer with methanol. Styrene-butadiene block copolymer is contained in the polymer. Since the viscosity increases with cooling, size distribution is considered to depend significantly on the viscosity rather than the rotational speed.

Experiments were conducted in which the viscosity was varied during the bulk polymerization by changing the amount of the chain transfer agent ( $t$-DDM). Rubber particle size distributions in bulk polymerization of run 5 ( $t$-DDM; $144 \mathrm{~g}$ ) are shown in Figure 2a. Large particles of more than $1 \mu \mathrm{m}$ decreased with bulk polymerization and cooling. The small particles of about $0.4 \mu \mathrm{m}$ increased. Size distributions in bulk polymerization were exactly bimodal, and the distribution after cooling was almost monomodal. Rubber particle size distributions in bulk polymerization of run 6 ( $t$-DDM: $168 \mathrm{~g}$ ) are shown in Figure 2b. In runs 6 and 5 , large particles of more than $1 \mu \mathrm{m}$ decreased with bulk polymerization and cooling. However, size distribution after cooling was distinctly bimodal. Polymer content of runs 5-6 was almost the same (about 35\%), and the polymer content of before cooling and after cooling were unchanged. The difference in runs 5 and 6 is the quantity of the added $t$-DDM, and the viscosity of the polymer material is different. The viscosity of the bulk polymerization at the end (after cooling) was $14200 \mathrm{cP}$ (run 5) and $7920 \mathrm{cP}$ (run 6). Furthermore, the rubber particle size distribution was changed with runs 5 and 6 by cooling as well as runs $1-4$. Therefore, it was suggested that the size distribution could be controlled by controlling the viscosity by temperature and addition of the chain transfer agent like $t$-DDM. 

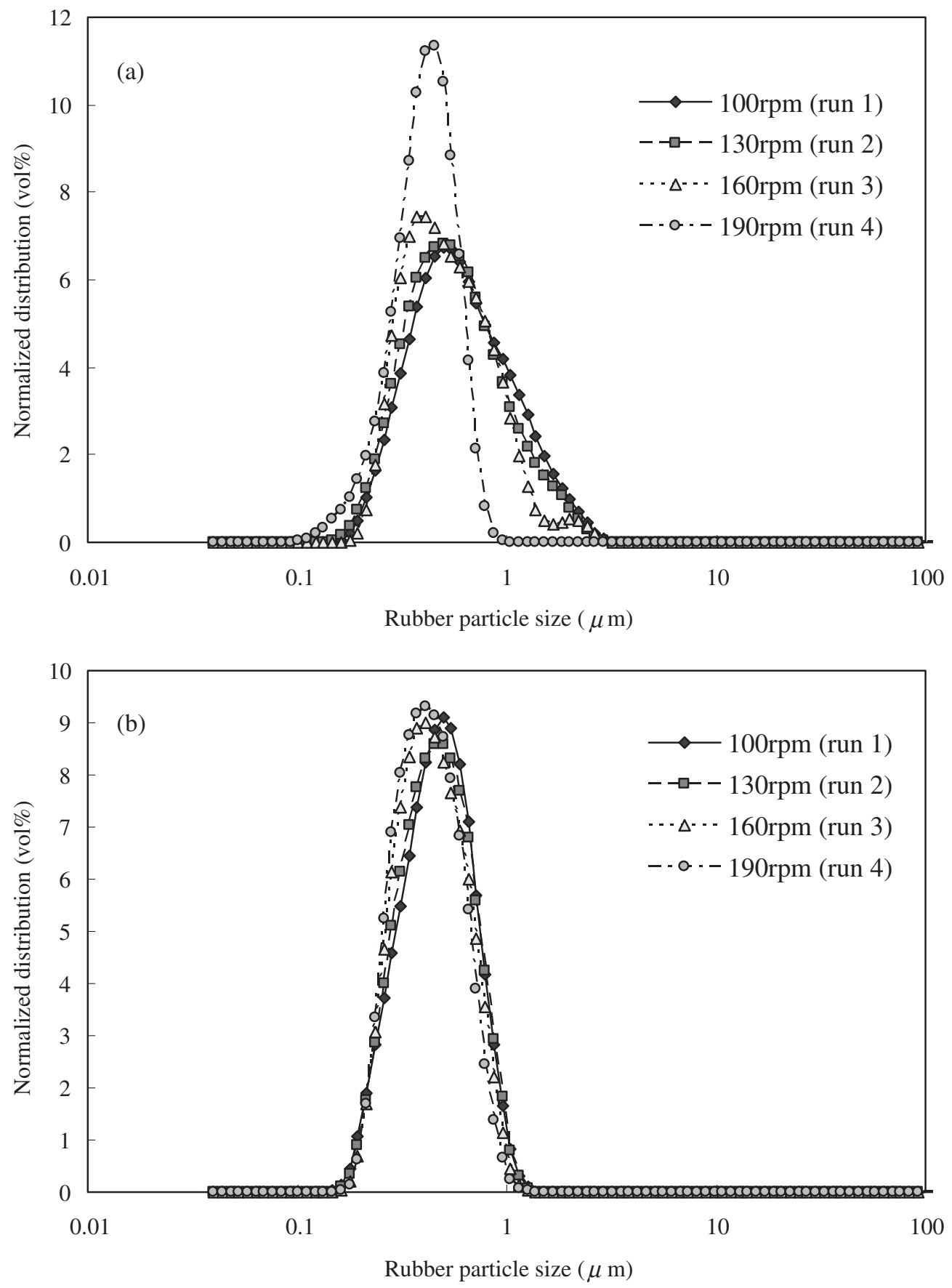

Figure 1. Distributions of rubber particle size in bulk polymerization: (a) before cooling (b) after cooling.

Rubber Particle Size Distribution by Control of Viscosity (run 7)

Size distribution by controlling the viscosity was studied in detail. Bulk polymerization and cooling were based on temperature as shown in Figure 3. Codes from A-1 to F-1 in Figure 3 are prepolymers sampled during the bulk polymerization and cooling. The suffix number 1 of the code means prepolymer.

The results are shown in Table I for viscosity and particle size of prepolymers, and in Figure 4 for size distribution. Phase inversion of $\mathrm{A}$ and $\mathrm{B}$ was not enough because polymer content was low, and particle size was not measurable because they didn't dissolve in the solvent. In run 7 as well as in run 5 and run 6 , large particles were reduced, but small particles increased during the bulk polymerization and the cooling process. Size distributions of D-1 and E-1 were bimodal.

\section{Change in Shape of Rubber Particle Sizes}

TEM photographs from A-1 to F-1 are shown in Figure 5. Capsule or onion-like small particles and onion-like large particles were observed in the TEM photographs of D-1 and E-1 with bimodal distributions. Onion-like large particles were observed in the TEM photographs of A-1 and B-1 with monomo- 

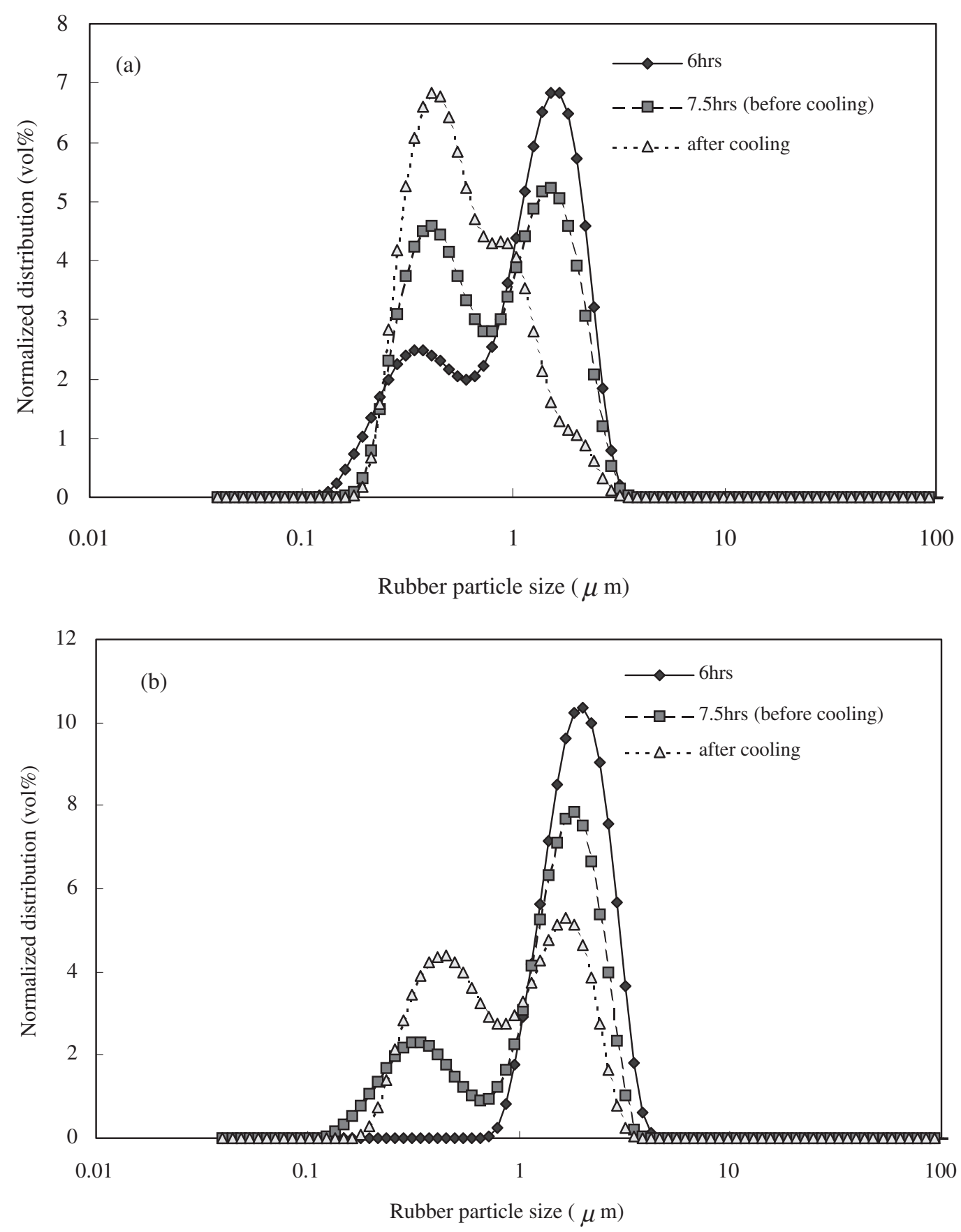

Figure 2. Distributions of rubber particle size in bulk polymerization: (a) run $5 ; t$-DDM $=144 \mathrm{~g}(0.18 \%)(\mathrm{b})$ run $6 ; t$-DDM $=168 \mathrm{~g}$ $(0.21 \%)$.

dal rubber particle size distribution. Shapes of the onion-like large particles of A-1 and B-1 were different from those of D-1 and E-1, and the inside structure of the onion-like large particles of A-1 and B-1 was comparatively sparse. Fingerprint-like particles were observed in the TEM photograph of C-1, which showed that the particles had agglomerated.

When polymerization using the prepolymer (D-1 and E-1) with bimodal distribution is carried out in the non-shearing state, it is assumed that the MBS resin with bimodal distribution is directly obtained.

\section{Mechanism of Bimodal Distribution}

The mechanism of formation of the bimodal distribution is considered as follows based on the TEM photographs.

Large rubber particles are formed after phase inversion, and become small by shearing. There are two contentions that the bimodal distribution is formed. First contention (1) is the increase in the fraction of small rubber particles. Large rubber particles change to small rubber particles by shearing, but small rubber particles do not become smaller than about $0.4 \mu \mathrm{m}$. Second contention (2) is the delay in changing 


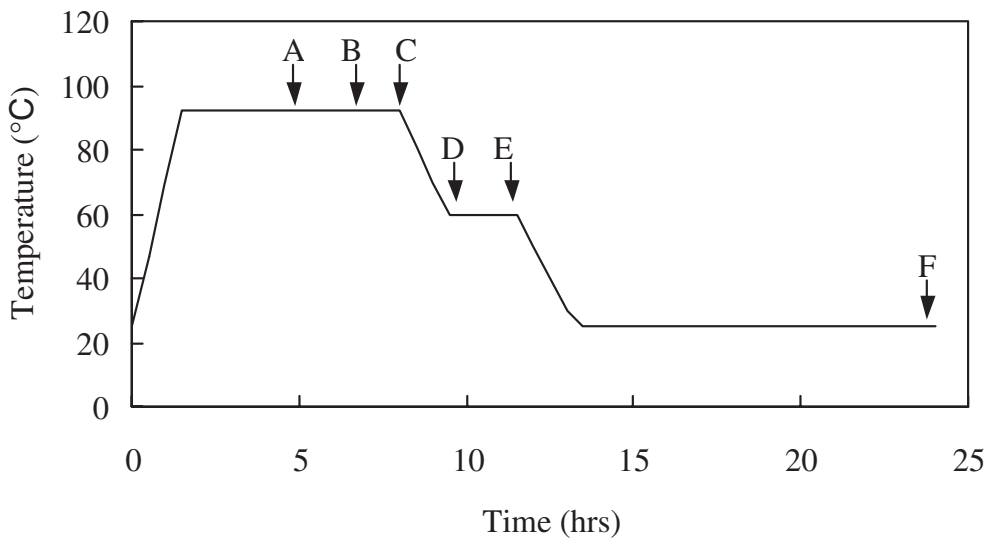

Figure 3. Temperature profile of run 7; A-F are sampled times of prepolymers.

Table I. Viscosity and rubber particle size of prepolymers

\begin{tabular}{|c|c|c|c|c|c|}
\hline \multirow{2}{*}{ Code } & Sampled temperature & Sampled time & Viscosity & Polymer content & Average rubber particle size \\
\hline & ${ }^{\circ} \mathrm{C}$ & hrs & $\mathrm{cP}$ & $\%$ & $\mu \mathrm{m}$ \\
\hline A-1 & 92 & 4.5 & 8300 & 26.9 & - \\
\hline B-1 & 92 & 6.5 & 10700 & 32.3 & - \\
\hline $\mathrm{C}-1$ & 92 & 8 & 12000 & 34.3 & 1.30 \\
\hline D-1 & 60 & 9.5 & 23500 & 36.2 & 1.15 \\
\hline E-1 & 60 & 11.5 & 23500 & 36.2 & 1.07 \\
\hline $\mathrm{F}-1$ & 25 & 24 & 44400 & 36.2 & 0.59 \\
\hline
\end{tabular}

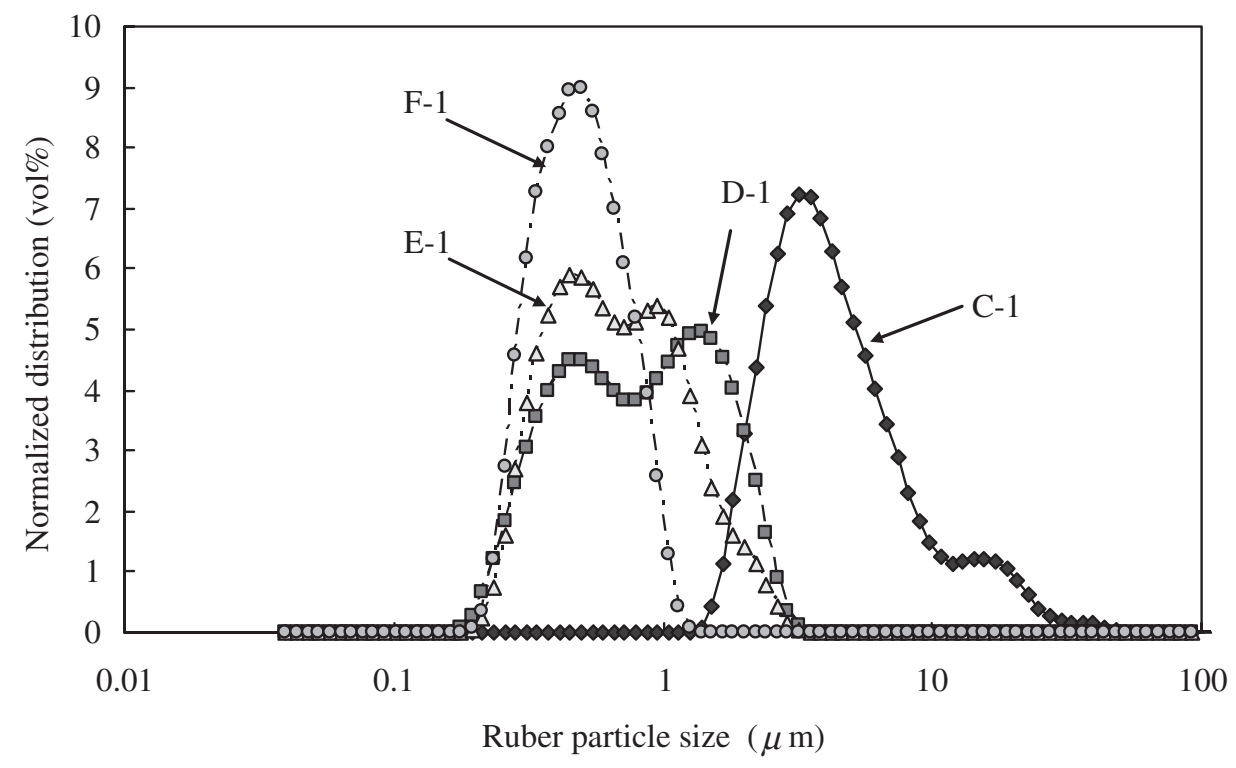

Figure 4. Distribution of rubber particle size in bulk polymerization of run 7.

to small particles. Large particles with compact inside structure like fingerprint were gradually changed to small particles, and which had a sparse inside structure like onion were rapidly changed to small particles.

The distribution of rubber particle sizes may be bimodal according to (1) and (2) in the transition period. It is thought that the bimodal distribution is signifi- cantly related to the inside 'onion' structure of rubber particles. Morita et al. explained the inside structure of rubber particles based on differences in the solubility parameters of matrix copolymer and styrene-butadiene copolymer. ${ }^{9}$ Therefore, possibly, the distribution of rubber particles must be also controlled by the composition of matrix copolymer and styrene-butadiene copolymer, and the matter needs further study. 

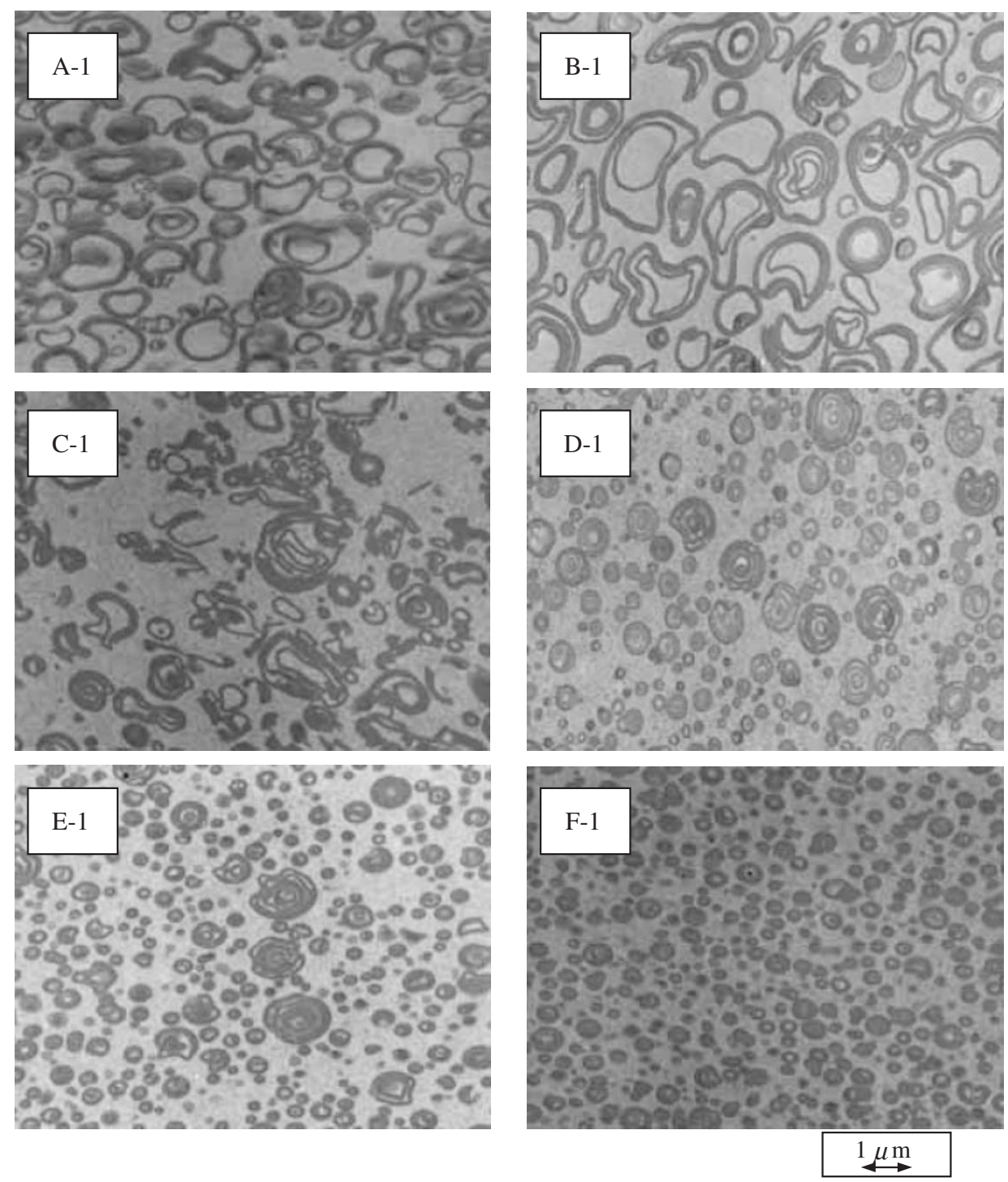

Figure 5. TEM photographs of prepolymers A-1-F-1.

Table II. Properties of MBS resins

\begin{tabular}{|c|c|c|c|}
\hline \multirow[t]{2}{*}{ Code } & $\begin{array}{l}\text { Charpy notched } \\
\text { impact strength }\end{array}$ & Haze & $\begin{array}{c}\text { Average rubber } \\
\text { particle size }\end{array}$ \\
\hline & $\mathrm{kJ} / \mathrm{m}^{2}$ & $\%$ & $\mu \mathrm{m}$ \\
\hline A-2 & 9.6 & 44.7 & 3.36 \\
\hline B-2 & 10.0 & 10.9 & 1.14 \\
\hline $\mathrm{C}-2$ & 9.6 & 13.0 & 1.54 \\
\hline $\mathrm{D}-2$ & 10.5 & 2.0 & 0.87 \\
\hline E-2 & 8.1 & 1.6 & 0.76 \\
\hline $\mathrm{F}-2$ & 2.9 & 1.1 & 0.55 \\
\hline
\end{tabular}

\section{Mechanical Properties}

Suspension polymerization was carried out using prepolymers from A-1 to F-1, and the mechanical properties were measured. Table II lists the properties and Figure 6 shows the rubber particles size distributions and Figures 7 are the TEM photographs of the pellets. The suffix number 2 of the code means MBS resin.
Rubber particle size of the pellets (Figure 6) became slightly smaller than the particle size of the prepolymer (Figure 4). The shapes of rubber particle of the pellets (Figure 7) were a little different from that of the prepolymer (Figure 5). It is possibly due to shearing at the time of suspension polymerization and extrusion. E-2 had monomodal distribution with a shoulder, D-2 showed bimodal distribution. We succeeded in getting the MBS resin with bimodal distribution without blending two resins or two prepolymers with different particle sizes.

Weight average molecular weight of A-2-F-2 was almost the same (about 340,000) and molecular weight distribution was also almost the same (about 2.4). Weight average molecular weight and molecular weight distribution were measured by gel permeation chromatography.

The relationship between the average rubber particle size and Charpy notched impact strength shown in Figure 8 indicated that the impact strength depend- 


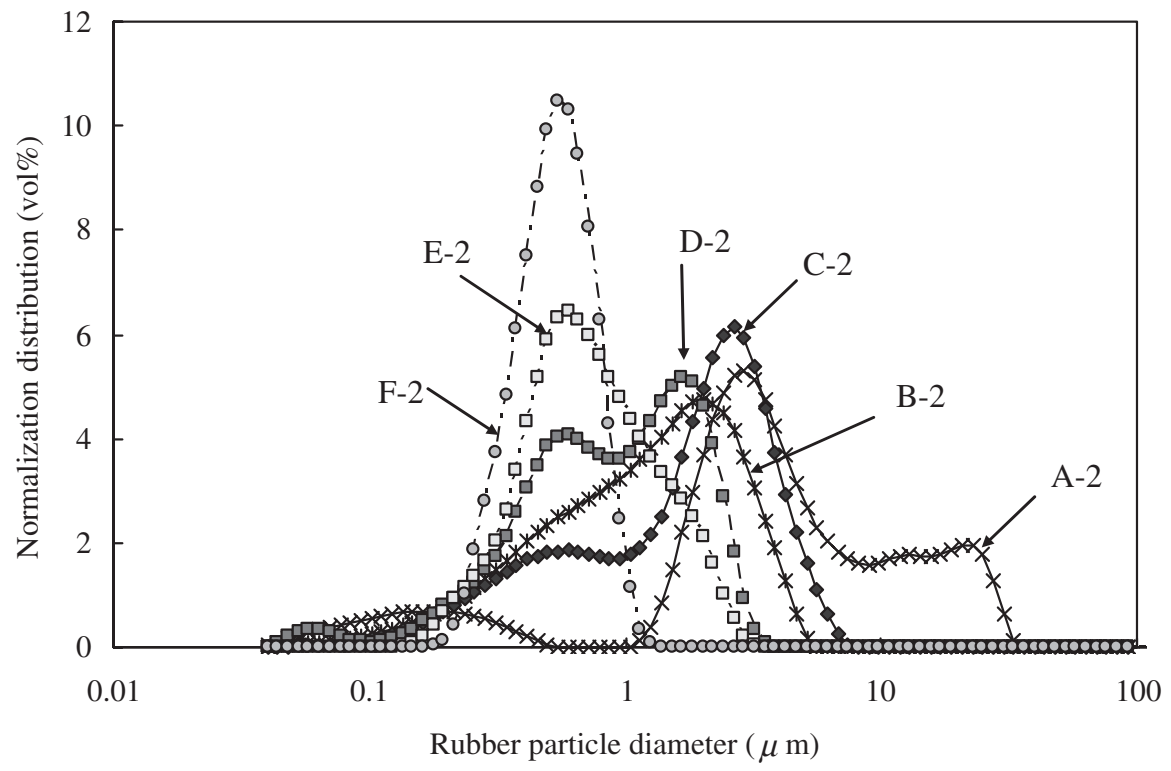

Figure 6. Distribution of rubber particle size of pellets A-2-F-2.
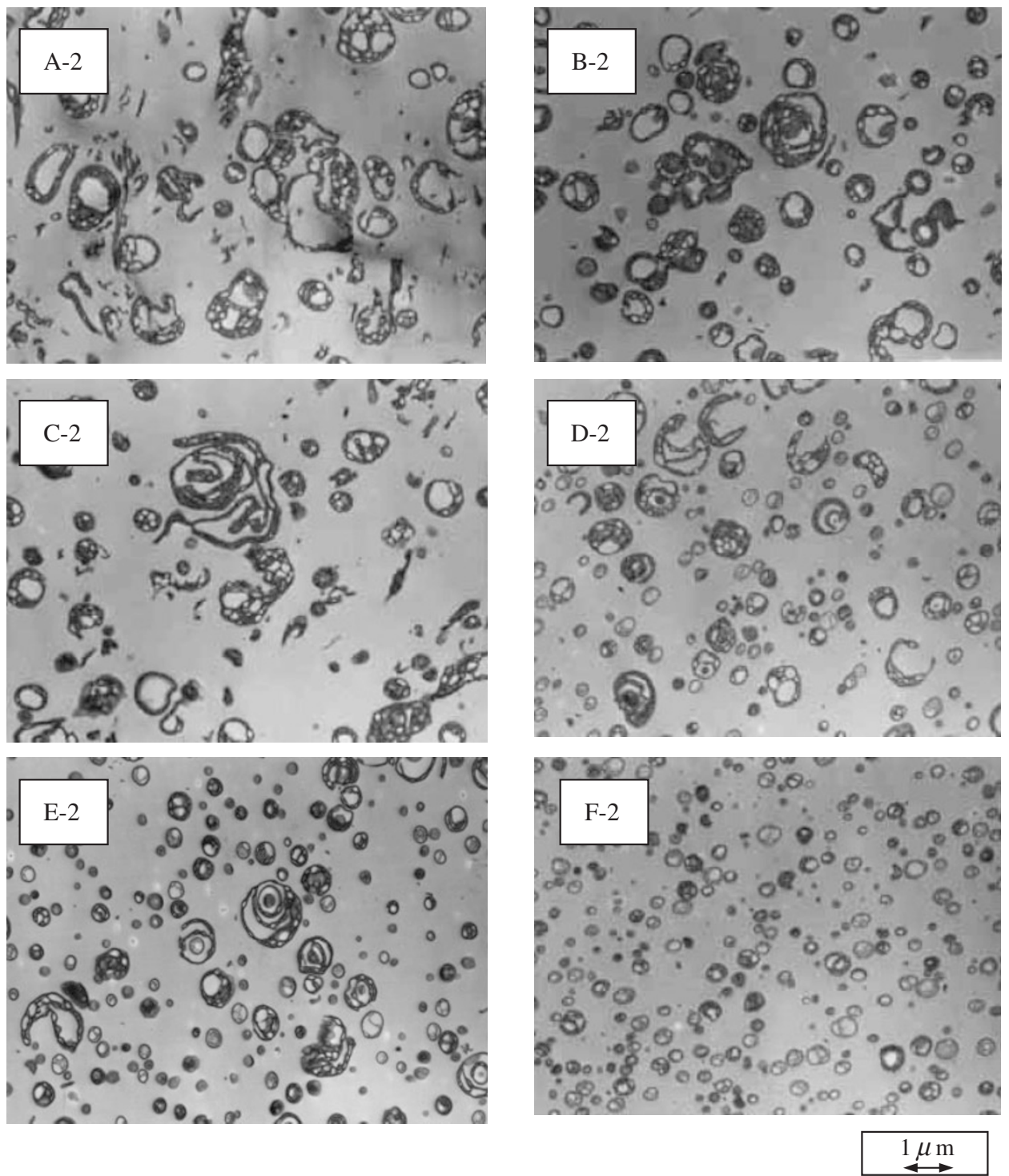

Figure 7. TEM photographs of pellets A-2-F-2. 


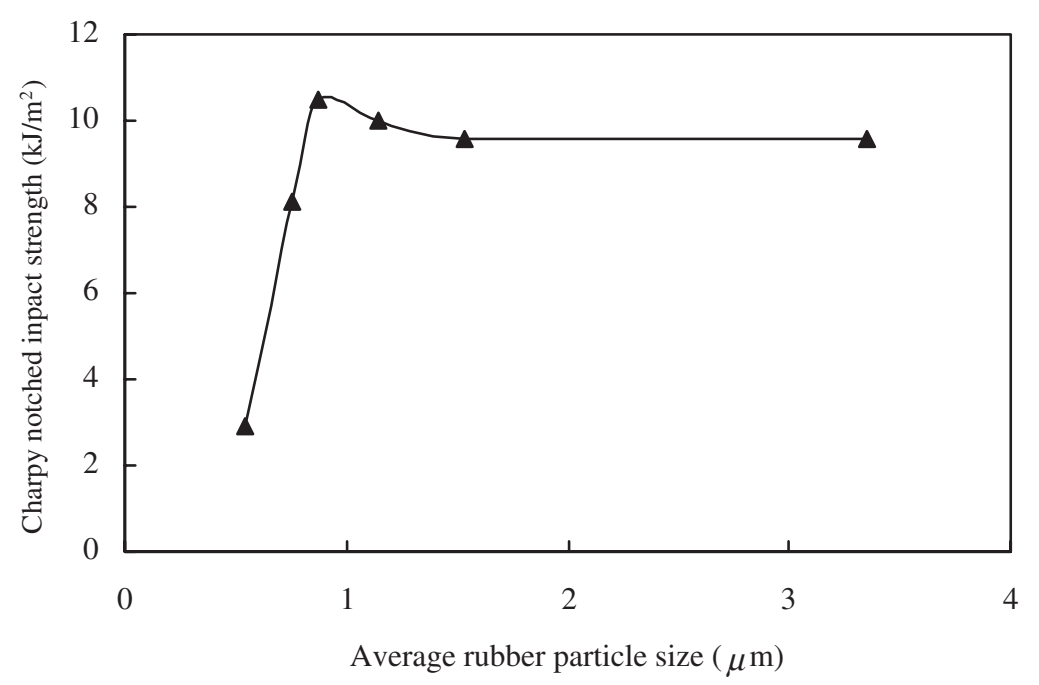

Figure 8. Relationship between average rubber particle size and Charpy notched impact strength.

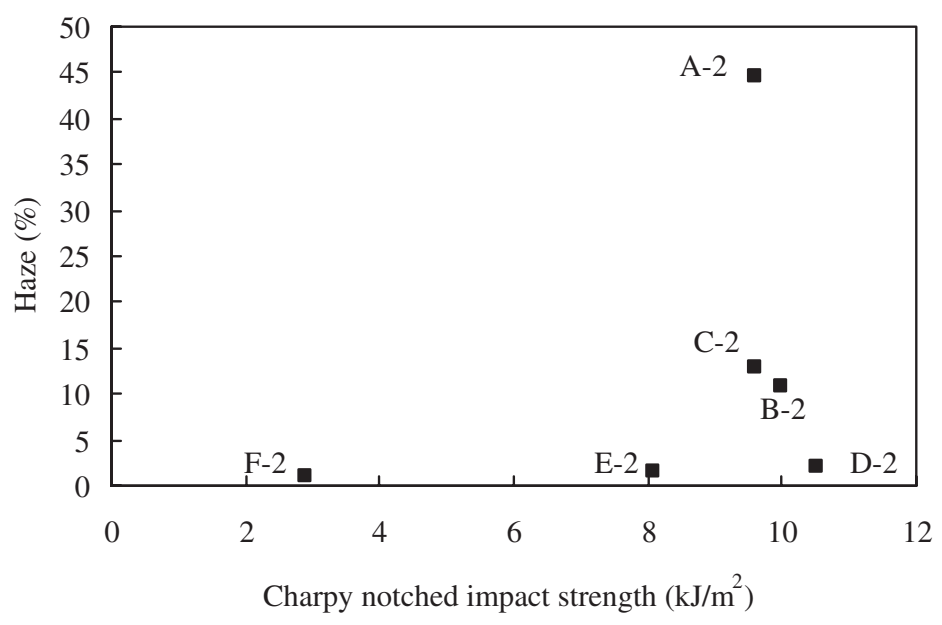

Figure 9. Relationship between Charpy notched impact strength and haze.

ed on the size when less than $0.87 \mu \mathrm{m}$, and the impact strength had a fixed value when size was greater than $0.87 \mu \mathrm{m}$.

Relations between Charpy notched impact strength and haze are shown in Figure 9. D-2 with a bimodal distribution obtained by this method had high impact resistance and good transparency. We have already reported properties of MBS resin with a bimodal distribution obtained by blending two resins. ${ }^{3} \mathrm{MBS}$ resin with bimodal distribution obtained by blending two resins has good balance of impact resistance and transparency. D-2 with the bimodal distribution obtained without blending had good balance as well as bimodal MBS resin obtained by blending.

\section{CONCLUSIONS}

The results of this study are summarized as follows: (1) Bimodal distribution of rubber particle sizes was observed during the bulk polymerization and cooling.
(2) The distribution of the rubber particle size could be controlled by viscosity or shearing.

(3) The mechanism of formation of the bimodal distribution is as follows: the increase in the fraction of small rubber particles and delay in changing to small rubber particles.

(4) MBS resin with a bimodal distribution was obtained without blending by carrying out a suspension polymerization using prepolymer with a bimodal distribution.

(5) MBS resin with a bimodal distribution obtained by this process was superior in balance of impact resistance and transparency.

\section{REFERENCES}

1. T. Gotou, I. Kishi, and S. Sakamaki, Jpn. Tokkyo Koho JP46 32748 (Sep. 23, 1971).

2. S. Kato, T. Hara, and I. Kawase, Jpn. Kokai Tokkyo Koho JP62 86046 (Apr. 20, 1987). 
In Situ Polymerization and Properties of Methyl Methacrylate-Butadiene-Styrene Resin with Bimodal Rubber Particle Size Distribution

3. J. Takahashi, H. Watanabe, J. Nakamoto, and T. Ito, Kobunshi Ronbunshu, 59, 527 (2002).

4. A. Echte and H. Gausepohl, U.S.Patent 4493922 (Jan. 15, 1985).

5. Y. Okamoto and T. Uno, U.S.Patent 4990569 (Feb. 5, 1991).

6. Y. Okamoto, H. Miyagi, and M. Kakugo, Macromolecules,
24, 5639 (1991).

7. S. Y. Hobbs, Polym. Eng. Sci., 26, 74 (1986).

8. B. Bile, J. M. Gloaguen, J. M. Lefebvre, and J. P. Tancrez, Plast., Rubber Compos., 30, 77 (2001).

9. T. Morita and J. Matsuo, Kobunshi Kakou, 45, 21 (1996). 